\title{
Modelo de fracionamento isotópico da água na bacia Amazônica (Primeira aproximaçào)
}

\author{
Attilio Dall'Olio $\left({ }^{\circ}\right)$; Eneas Salati $\left({ }^{* *}\right)$; Carlomam Tatagiba de Azevedo $\left({ }^{* * *}\right)$; Eiichi Matsui $\left({ }^{* *}\right)$
}

\section{Resumo}

Neste trabalho são apresentados dois modelos de fracionamento isotópico da água. No primeiro admite-se como única fonte de vapor dágua o oceano Atlântico, sendo o vapor introduzido na região Amazônica através dos ventos predominantes do quadrante Este. Em seguida, é apresentado outro modelo admitindo-se que a floresta atua como uma segunda fonte de vapor dágua, sendo a sua contribuiçāo, para a produção das chuvas da região, da mesma ordem de grandeza que a do vapor proveniente do oceano.

\section{INTRODUÇÃo}

Os trabaihos preliminares de Salati et al. (1979) demonstram haver uma variaçäo nas concentrações de 180 e D nas águas dos rios da bacia Amazônica. As primeiras aplicações práticas desta variabilidade foram feitas por Matsui et al. (1972 e 1976) para determinar as vazões relativas dos rios Negro e Solimões e por Reis et al. (1977) na identificação das águas da orla da ilha de Marajó.

As diferentes concentrações de 180 e D nas águas dos afluentes do Amazonas, demonstraram ainda, a variabilidade desses isótopos nas chuvas de diversas sub-regiōes. Como a concentração isotópica da chuva depende, entre outros fatores, da origem do vapor dágua, ficou evidenciada a possibilidade de se utilizar esta metodologia no estudo da dinâmica do vapor dágua da regiăo.

O problema a ser abordado é a orıgem primária do vapor dágua envolvido na formação das nuvens e chuvas e a eventual influência do vapor oriundo da própria região. Em outras palavras, procura-se estimar a reciclagem da água da região ou seja, a influência da fioresta no ciclo da água através da transpiração.
Em outro trabalho (Salati et al., 1979) são analisados os dados isotópicos obtidos em dois anos de observação e é feita uma análise desse modelo simplificado.

\section{MATERIAL E MÉtodo}

Neste trabalho foram utilizados os seguintes dados:

Composição isotópica das águas de chuva coletadas nos postos meteorológicos de Belém, Cachoeira do Arari, São Sebastião, Santa Cruz do Arari, Amapá, Taperinha, Manaus, Uaupés e Benjamin Constant.

As amostras foram preparadas coletandose toda a água do pluviômetro para se obter uma média mensal ponderada. As concentrações de 180 foram determinadas por espectrometria de massa, sendo os resultados expressos em desvios relativos ao padrão V-SMOW (Gonfiantini, 1978), segundo a expressão:

$$
\$ 180=\frac{(180 / 160) \text { amostra }}{(180 / 160) \text { V-SMOW }}-1 \quad 10^{3}
$$

Os dados obtidos enconram-se na tabela 1.

Valores médios mensais do fluxo de vapor dágua oceânico $\left(\mathrm{O}_{0}\right)$ que penetra na região. Nestes cálculos foram utilizados os valores de radios-sondagens de Belém. Foram computados por integração numérica, a partir dos valores diários de umidade específica e da velocidade, desde o nível do solo até o nível de 500 milibares $(\simeq 5.850 \mathrm{~m}$ de altitude). Estes dados foram fornecidos por Marques (comunicação pessoal) (Tabela 2).

\footnotetext{
(*) - Centro de Energia Nuclear da Universidade Federal de Pernambuco, Recife.

(*) - Instituto Nacional de Pesquisas da Amazônia, Manaus.

(***) - Centro Técnico Aeroespacial.
} 
As temperaturas médias mensais $T$, ao nivel dos 850 milibares, correspondentes à camada mediana da distribuição do vapor dágua. isto é, aquela camada abaixo da qual se encon. tra aproximadamente $50 \%$ da massa total do vapor (Tabela 2)

Para fins computacionais, a faixa central da bacia Amazônica, limitada pelo Equador e pelo paralelo de $5^{\circ} \mathrm{Sul}$, e pelos meridianos $48^{\circ} 30^{\prime}$ e $72^{\circ} 30^{\prime}$ Oeste foi subdividida em 8 setores de $3^{\circ}$ de longitude e numerados de 1 a 8 no sentido Leste-Oeste (Fig. 1).

Os totais de precipitação $\mathrm{Pn}_{\mathrm{n}}$, para cada setor, computados por integração gráfica das isoietas mensais (Tabela 3) .

Os valores médios da evapotranspiração $E_{n}$. calculados pelo método de Penmann adaptado a áreas cobertas por florestas; estes valores foram cbtidos por Villa Nova et al. (1976). Neste trabalho foram utilizados os valores médios de $E$ de localidades incluídas em cada setor e não os valores específicos de 1974 (Tabela 3).

\section{TEORIA DO MÉTODO}

As espécies moleculares da água, $\mathrm{H}_{2}{ }^{16} \mathrm{O} \mathrm{e}$ $\mathrm{H}_{2}{ }^{18} \mathrm{o}$, possuem tensões de vapor diferentes; por isso, durante os processos de evaporação e condensação, verifica-se um fracionamento isotópico, isto é, as concentrações relativas dessas espécies moleculares, nas fases vapor e líquida, são diferentes.

O coeficiente de fracionamento isotópico $\propto$. definido pela expressão :

$$
\propto=\mathrm{R}_{\text {liquido }} / \mathrm{R} \text { vapor, }
$$

onde,

$$
R \propto \frac{\left[{ }^{18} 0\right]}{\left[{ }^{16} 0\right]},
$$

é a relação entre as concentrações das duas espécies isotópicas de interesse ou seja a concentraçãc isotópica relativa. O coeficiente $\propto$ depende só da temperatura, quando a transic̣ão de fase se processa em condições de equilíbrio.

Nos processos naturais o valor de $\propto$ pode afastar-se do valor de equilíbrio pela influência de fatores cinéticos; contudo, a variabilidade da cơmposição isotópica das massas de água da hidrosfera pode, ainda, ser significativamente analisada, comparando os dados experimentais com os valores fornecidos por modelos teóricos que postulem condições de equilibrio. Em particular, análises deste tipo são adequadios quando são tomados em consideração valores médios ponderados ao longo de períodos de tempo relativamente longos, ou para descrever as caracteristicas gerais do ciclo meteorológico da água de regiões bastante extensas. Os modelos de equilíbrio são, de outro lado, inadequados para a análise de eventos meteorológicos individuais, como precipitações isoladas.

O fator de fracionamento, $\propto$, fornece diretamente a relação das concentrações isotópicas relativas, entre as duas fases, quando são verificadas as seguintes hipóteses:

I. a transição de fase é isotérmica quase-estática;

II. o equilíbrio termodinâmico estende-se a todo o sistema;

III. a massa de água da fase primáriá pode ser considerada como infinita.

Este modelo pode, por exemplo, ser aplicado à evaporação dos oceanos, dado que a massa total de vapor formada é desprezivel, reiativamente à massa de água dos próprios oceanos.

Considerando-se, de outro lado, a processo de condensação que dá origem às precipitações, verifica-se que a terceira hipótese não é adequada, pois que a massa de vapor que condensa é, em geral, uma fração apreciável da massa total da fase primária.

Neste caso, a relação entre as concentraçōes isotópicas é caracterizável pelo enriquecimento isotópico relativo, $\beta_{\mathrm{C}}$, que representa a relação entre as concentrações isotópicas relativas da massa condensada e a do vapor inicial dado pela expressẫo:

$$
\beta_{C}=\frac{\propto}{(1-F) \propto+F}
$$

onde $\propto$ é o fator de fracionamento já definido e $F$ é a fração residual da massa de vapor; a expressăo (2) é obtida considerando o siste- 
TABELA 1 - Precipitaçōes médias mensais e dados isotópicos médios mensais de águas de chuva dos postos de observação localizados ao longo da faixa cen. tral da bacia Amazônica no ano de 1974. Foram incluídos os dados de UAÚPÉS, embora esteja um pouco ao norte da área central.

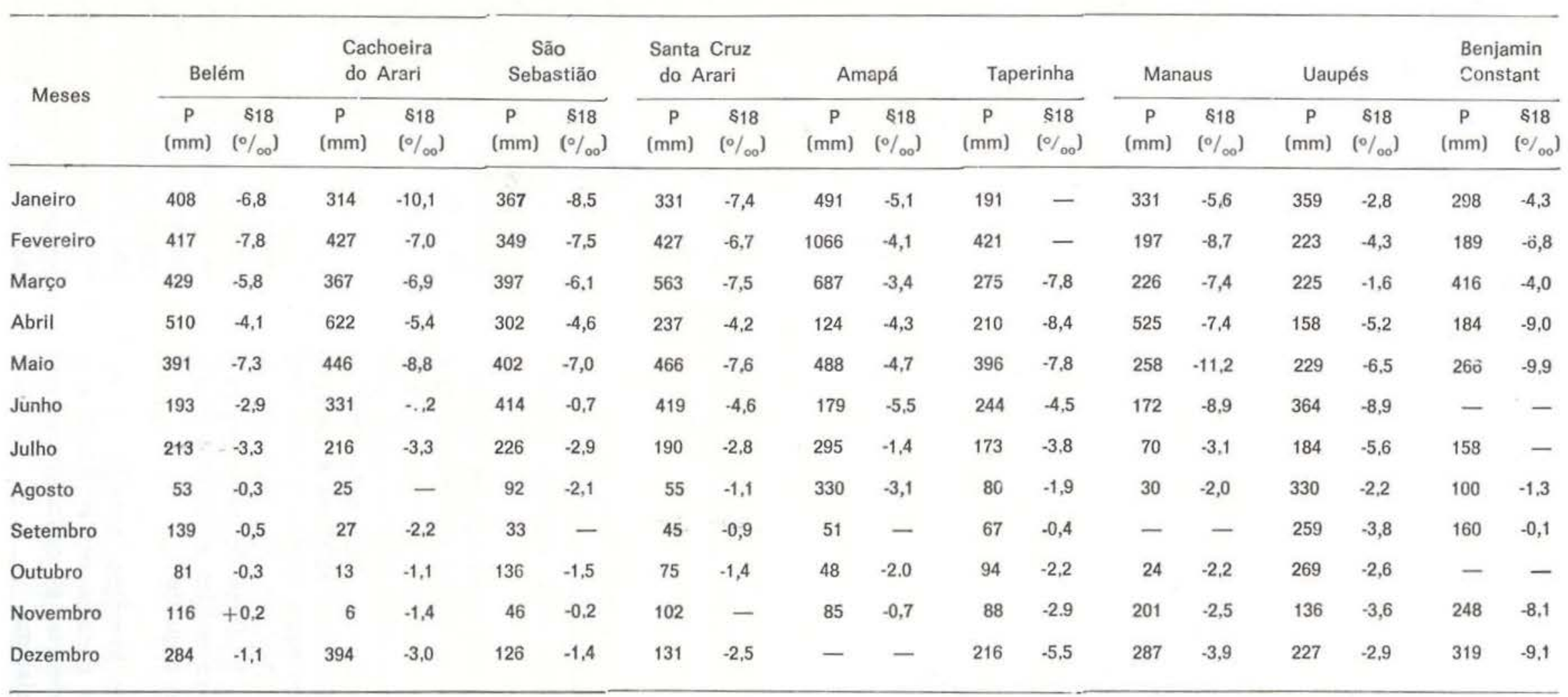




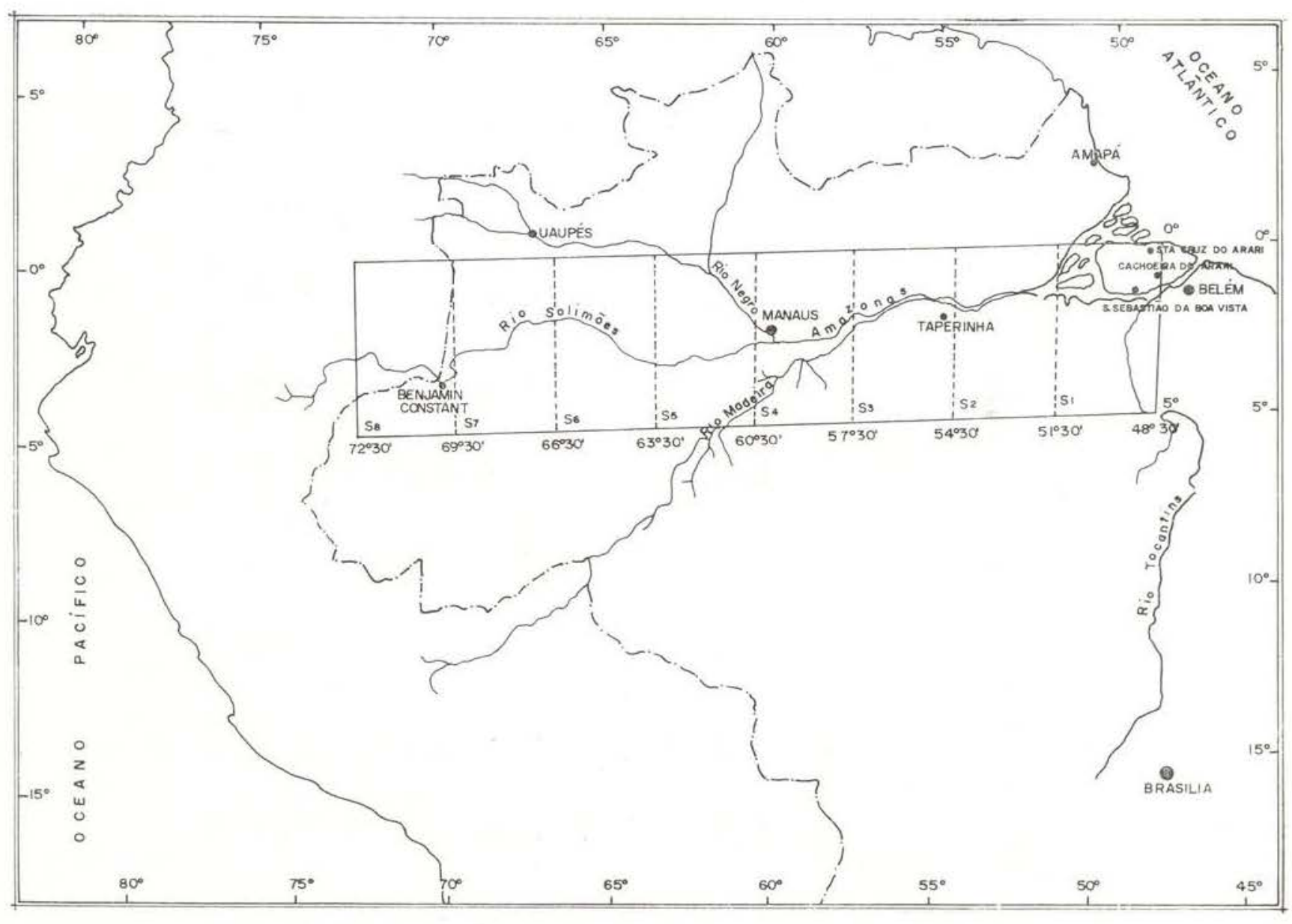

Fig. 1 - Faixa central da Bacia Amazônica dividida em oito setores.

ma como fechado e aplicando, portanto, o princípio da conservação de massa para a água total e para cada espécie isotópica (Dall'Olio, 1976) .

O fenômeno das precipitações consiste, como o próprio nome indica, na remoçãa (precipitação) do condensado e, portanto, também a segunda hipótese não é mais adequacia para representar o fenômeno. Para determinar as concentrações isotópicas relativas é necessário recorrer a um outro modelo teórico, chamado modelo de Rayleigh, que, mantendo somente a primeira hipótese de que o processe é isotérmico quase-estático substitui a segun. da hipótese pela seguinte:

II'. a fase em formação, considerada instante por instante em equilíbrio com a fase primária, é continuamente retirada do sistema.
Aplicando-se as mesmas considerações sobre a conservação da massa total e de cada espécie isotópica a quantidades infinite. simais da fase em formação e integrando, obtém-se o enriquecimento isotópico relativo $\beta^{\prime} \mathrm{C}$,

$$
\beta_{C}^{\prime}=\frac{1-F^{\propto}}{1-F}
$$

onde os símbolos já foram definidos. O modelo de Rayleigh pode ser aplicado também a processos quase-estáticos não-isotérmicos; neste caso, quando da integração, será necessário considerar a variação do fator de fracio. namento, $\propto$, com a temperatura.

Além das expressões (2) e (3), que fornecem o enriquecimento isotópico relativo para a fase em formação, é possível determi- 
nar o enriquecimento isotópico relativo da fração residual do vapor a partir das relaçōes

$$
\beta_{v}=\frac{1}{(1-F) \propto+F}, \ldots \ldots . .
$$

para o modelo de fases em equilíbrio num sistema fechado e

$$
\beta_{v}^{\prime}=\frac{F^{\propto}}{F} \ldots \ldots \ldots \ldots \ldots \ldots .
$$

para o modelo de Rayleigh,

O modelo de Rayleigh isotérmico pode ser utilizado para descrever a variação da composição isotópica média mensal das precipitações ao longo da bacia Amazônica tace à notável constância dos valores médios, espacial e temporal, tanto da temperatura como da umidade relativa.

A fase primária será constiłuída pela massa de vapor da qual originam-se as precipitaçōes. As determinaçōes experimentais (Marques et al., 1977) mostram que a fonte primária de vapor é constituída pelo vapor originado no oceano Atlântico e transportado ao longo da bacia pelc fluxo zonal, no sentido de Leste para Oeste.

Portanto, em primeira aproximação, podese aplicar o modelo de Rayleigh tomando em consideração somente essa fonte de vapor;

TABELA 2 - Massas de água (em $10^{17} \mathrm{~g}$ ) mensais do fluxo de vapor $Q_{o}$, das precipitaçóes $P$ e da evapotranspiração $\mathrm{E}$; e temperatura média mensal $\mathrm{T}$, em ${ }^{\circ} \mathrm{C}$, da ca. mada mediana da coluna de vapor.

\begin{tabular}{lcccc}
\hline Mês & $Q_{\circ}$ & $P$ & $E$ & $T$ \\
\hline JAN & 4,73 & 3,91 & 1,67 & 17,6 \\
FEV & 4,13 & 4,36 & 1,67 & 18,0 \\
MAR & 3,12 & 4,42 & 1,62 & 17,3 \\
ABR & 3,45 & 4,31 & 1,53 & 17,5 \\
MAI & 3,97 & 4,59 & 1,54 & 17,1 \\
JUN & 3,99 & 3,76 & 1,50 & 17,7 \\
JUL & 3,70 & 2,32 & 1,62 & 17,0 \\
AGO & 3,74 & 1,58 & 1,82 & 18,0 \\
SET & 3,99 & 2,07 & 1,97 & 18,0 \\
OUT & 3,75 & 1,68 & 2,00 & 18,3 \\
NOV & 4,05 & 2,16 & 1,86 & 18,5 \\
DEZ & 4,36 & 3,43 & 1,73 & 18,4 \\
\hline
\end{tabular}

contudo, observando que, nos primeiros cinco meses do ano considerado (1974), a massa total das precipitações é superior, ou quase igual, à massa totai do vapor oceânico que penetra na Bacia (Tabela 2), será sucessivamente necessário acrescentar ao modelo a contribuição de, pelo menos, uma outra fonte de vapor, que será identificada com o processo de evapotranspiração da floresta ao longo da bacia.

MODELO DE RAYLEIGH ISOTÉRMICO PARA UMA ÚNICA FONTE

Para determinar a composição isotópica das precipitaçōes ao longo da bacia Aınazônica pode ser utilizado o esquema representado na Figura 2. As precipitações $\mathrm{Pn}$, do setor genérico $\mathrm{S}_{\mathrm{n}}$, originam-se da massa de vapor $\mathrm{O}_{\mathrm{n}}$ transportada pelo fluxo zonal e constituída pelo vapor residual do setor precedente, $s_{n}-1$.

TABELA 3 - Valores de P e E (em $\mathrm{mm} / \mathrm{mês}$ ) para os vários setores $S_{n}$.

\begin{tabular}{lrrrrrrrrr}
\hline & $\mathrm{S}_{1}$ & $\mathrm{~S}_{2}$ & $\mathrm{~S}_{3}$ & $\mathrm{~S}_{4}$ & $\mathrm{~S}_{5}$ & $\mathrm{~S}_{6}$ & $\mathrm{~S}_{7}$ & $\mathrm{~S}_{8}$ & \\
\hline $\mathrm{P}$ & 338 & 367 & 261 & 162 & 240 & 227 & 294 & 327 & $\mathrm{~J}$ \\
$\mathrm{E}$ & 130 & 112 & 127 & 121 & 121 & 127 & 124 & 188 & \\
$\mathrm{P}$ & 329 & 391 & 389 & 377 & 234 & 224 & 248 & 283 & $\mathrm{~F}$ \\
$\mathrm{E}$ & 115 & 98 & 109 & 109 & 115 & 115 & 109 & 112 & \\
$\mathrm{P}$ & 414 & 503 & 462 & 300 & 191 & 152 & 157 & 320 & $\mathrm{M}$ \\
$\mathrm{E}$ & 118 & 102 & 118 & 118 & 127 & 124 & 121 & 121 & \\
$\mathrm{P}$ & 305 & 357 & 308 & 238 & 295 & 321 & 318 & 303 & $\mathrm{~A}$ \\
$\mathrm{E}$ & 108 & 46 & 117 & 108 & 108 & 114 & 108 & 108 & \\
$\mathrm{P}$ & 355 & 399 & 310 & 331 & 309 & 249 & 274 & 377 & $\mathrm{M}$ \\
$\mathrm{E}$ & 112 & 105 & 115 & 121 & 112 & 115 & 109 & 102 & \\
$\mathrm{P}$ & 220 & 201 & 260 & 157 & 158 & 262 & 393 & 481 & $\mathrm{~J}$ \\
$\mathrm{E}$ & 120 & 114 & 117 & 123 & 108 & 102 & 99 & 96 & \\
$\mathrm{P}$ & 120 & 111 & 138 & 117 & 132 & 190 & 246 & 264 & $\mathrm{~J}$ \\
$\mathrm{E}$ & 133 & 105 & 133 & 143 & 121 & 112 & 105 & 99 & \\
$\mathrm{P}$ & 39 & 68 & 56 & 62 & 71 & 89 & 244 & 268 & $\mathrm{~A}$ \\
$\mathrm{E}$ & 143 & 130 & 149 & 158 & 133 & 124 & 118 & 112 & \\
$\mathrm{P}$ & 88 & 112 & 97 & 102 & 103 & 181 & 243 & 249 & $\mathrm{~S}$ \\
$\mathrm{E}$ & 168 & 129 & 153 & 156 & 138 & 132 & 126 & 114 & \\
$\mathrm{P}$ & 72 & 59 & 73 & 90 & 72 & 157 & 222 & 206 & $\mathrm{O}$ \\
$\mathrm{E}$ & 183 & 127 & 167 & 155 & 140 & 140 & 127 & 133 & \\
$\mathrm{P}$ & 78 & 71 & 102 & 126 & 153 & 237 & 250 & 207 & $\mathrm{~N}$ \\
$\mathrm{E}$ & 165 & 108 & 147 & 138 & 129 & 129 & 117 & 120 & \\
$\mathrm{P}$ & 225 & 242 & 221 & 277 & 232 & 227 & 231 & 293 & $\mathrm{D}$ \\
$\mathrm{E}$ & 143 & 112 & 140 & 127 & 124 & 130 & 118 & 121 & \\
\hline & & & & & & & & & \\
\hline & & & & & & & & &
\end{tabular}



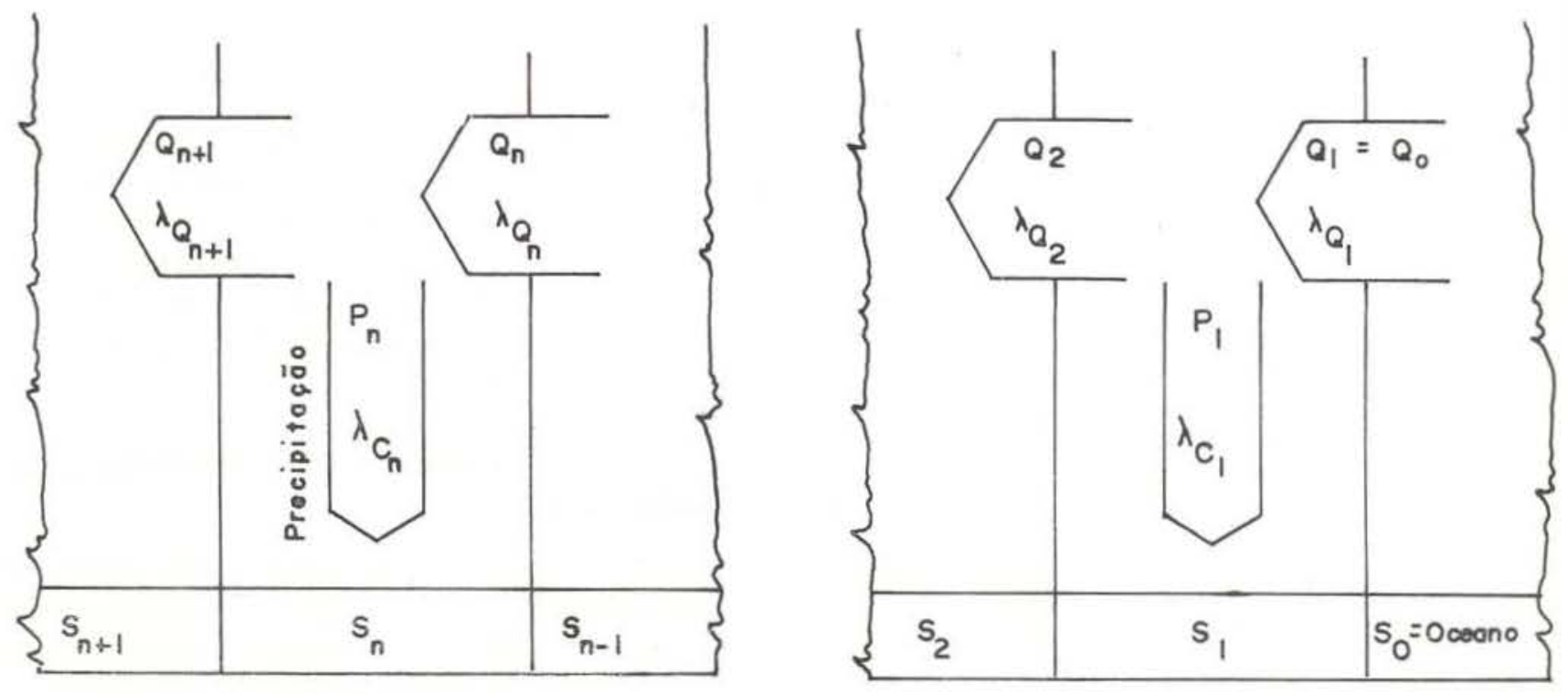

Fig. 2 - Modelo para o cálculo de $\lambda_{c_{n}}$ considerando como única fonte de vapor o oceano Atlântico.

Indicando com $\beta^{\circ} \mathrm{C}_{\mathrm{n}}$ O enriquecimento isotópico, relativo ao vapor $Q_{n}$, das precipitaçōes $P_{n}$ do setor $S_{n}$, tem-se por (3) que

$$
\beta_{\mathrm{Pn}_{\mathrm{n}}}^{\prime}=\frac{1-\mathrm{Fn}^{\propto}}{1-\mathrm{Fn}_{\mathrm{n}}} .
$$

onde $F_{\mathrm{n}}$ é a fração residual do vapor no setor $\mathrm{S}_{\mathrm{n}}$, estimada pela expressão,

$$
F_{n}=\frac{Q_{n}-P_{n}}{Q_{n}} \ldots \ldots \ldots \ldots \ldots .
$$

e, $\propto$, é o fator de fracionamento isotópico.

$O$ enriquecimento isotópico $\beta^{\prime}{ }_{a_{n+1}}$ sem. pre relativo à massa de vapor $Q_{n}$, do vapor residual $Q_{n+1}$ será, pela (5):

$$
\beta_{Q_{n+1}}^{\prime}=\frac{F_{n} \propto}{F_{n}} \ldots \ldots \ldots \ldots \ldots .
$$

Introduzindo o enriquecimento isotópico ac V-SMOW, definito como

$$
y=\frac{R_{\text {amostra }}}{R_{\text {V-SMOW }}}
$$

pode-se escrever :

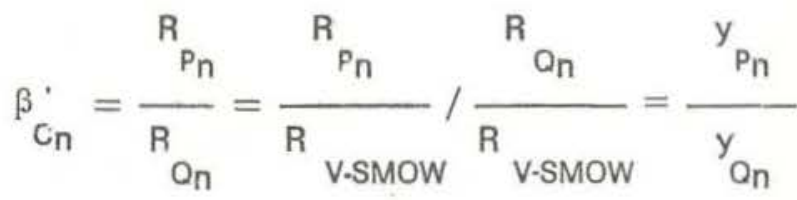

e as expressōes (6) e (7) resultam nas:

$$
\begin{aligned}
& y_{P_{n}}=\frac{1-F_{n}{ }^{\propto}}{1-F_{n}} y_{O_{n}} \ldots \ldots \ldots . . \\
& y_{a_{n+1}}=\frac{F_{n}}{F_{n}} y_{O_{n}} \ldots \ldots \ldots \ldots .
\end{aligned}
$$

As relações (6') e (7') permitem computar a composição isotópica das precipitaçōes para cada setor $S_{n}$ desde que sejam conhecidos:

i) a massa de vapor que entra na bacia;

ii) a composição isotópica inicial desse vapor;

iii) a temperatura média de condensação em cada setor $\mathrm{S}_{n}$;

iv) os correspondentes valores da precipitaçäo, $\mathrm{Pn}$. 
Como foi exposto no capítulo Material e Método, tais parâmetros só são disponíveis os totais das precipitações e a massa de vapor transportado pelo fluxo zonal dentro da bacia. É, portanto, necessário estimar, de modo adequado, os outros parâmetros a saber: as temperaturas de condensação e a composição isotópica do vapor oceânico.

Observando-se que durante cada mês, a temperatura e a umidade relativa apresentam uma notável constância, ao longo da faixa central da bacia, é razoável admitir-se uma única temperatura de condensação para todos os setores $S_{n}$ e admiti-la como sendo a própria temperatura média mensal da camada mediana da massa de vapor.

Quanto à estimativa da composição isotópica do vapor oceânico, pode-se observar que, para o setor $\mathbf{S}_{1}$, a expressão (6') pode ser escrita como :

$$
\mathrm{y}_{1}=\frac{1-F_{1}}{1-F_{1}{ }_{1}} y_{P_{1}}
$$

que fornece justamente o enriquecimento isotópico, relativo ao $\mathrm{V}$-SMOW da massa de vapor 01 que entra no setor $\mathrm{S}_{1} \mathrm{em}$ função do enriquecimento isotópico, relativo ao $\mathrm{V}$-SMOW, das precipitações $\mathrm{P}_{1}$ do mesmo setor. Mas, sendo $\mathrm{S}_{1}$ o setor mais oriental, a massa de vapor $\mathrm{Q}_{1}$ é o próprio vapor oceânico $Q_{0}$.

Para dispor de um valor suficientemente representativo de $y_{p_{i}}$ pode-se utilizar o valor médio ponderado de todas as localidades incluídas no setor $\mathbb{S}_{1}$, quais sejam São Sebastião, Santa Cruz do Arari na ilha de Marajó, Belém e Amapá.

\section{A CONTRIBUIÇÃO DA EVAPOTRANSPIRAÇÃO}

O modelo apresentado no subcapítulo precedente, não pode ser utilizado para interpretar os valores experimentais dos primeiros meses do ano considerado (1974), dado que a massa de vapor transportado pelo fluxo zonal é inferior à massa total das precipitações mesmo levando em consideração somente a faixa central considerada. É, portanto, necessário tomar em consideração, pelo menos. uma outra fonte de vapor.

O fluxo meridional de vapor, de origem oceânica ou continental, além de constituir uma reduzida fração do fluxo total, apresenta um balanço de massa praticamente nulo para a faixa central da bacia e não pode portanto compensar o deficit de vapor constado nos primeiros meses de 1974. Do ponto de vista meteorológica, não existem evidências de outras fontes externas de vapor, além das consideradas, que possam contribuir apreciavelmente ao fluxo de vapor ao longo da fiaxa central da bacia.

De outro lado a extensão e a densidacie da floresta amazônica, que cobre de modo compacto e contínuo a faixa central da bacia, sugerem quie a evapotranspiração pode constituir-se em uma fonte apreciável de vapor. Outros resultados preliminares confirmam que a evapotranspiração é um componente importante do balanço hídrico da bacia Amazônica (Vila Nova, 1976; Marques et al., 1977; Mollion, 1976) .

Para introduzir essa fonte de vapor no modelo computacional, pode-se utilizar o esquema representado na Figura 3 . As precipitações $P_{n}$ de um setor $S_{n}$ originam-se, desta vez, de uma massa de vapor Q'n composta pelo vapor $\mathrm{Q}_{\mathrm{n}-1}$ ' transportado pelo fluxo zonal e constituido do vapor residual do setor precedente $\mathrm{S}_{\mathrm{n}-1}$ ' e pelo vapor $\mathrm{En}_{\mathrm{n}}$ fornecido pela evapotranspiração da fioresta no próprio setor $S_{n}$.

$O$ enriquecimento isotópico y $\mathrm{O}_{\mathrm{n}}$ J, relativo ao V-SMOW, da massa de vapor Q'n, pode ser estimado pela média ponderada dos enriquecimentos isotópicos, relativos ao $\mathrm{V}$-SMOW. da massa de vapor $Q_{n-1}$ y $\mathrm{Q}_{n-1}$, e da massa de vapor $E_{n}, y_{E_{n}}$; isto é :

$$
y_{Q_{n}}=\frac{Q_{n-1} y_{n-1}+E_{n E_{n}}}{O_{n-1}+E_{n}} \ldots . .
$$

onde está implícita a hipótese que as duas massas de vapor formam uma mistura homogênea. 

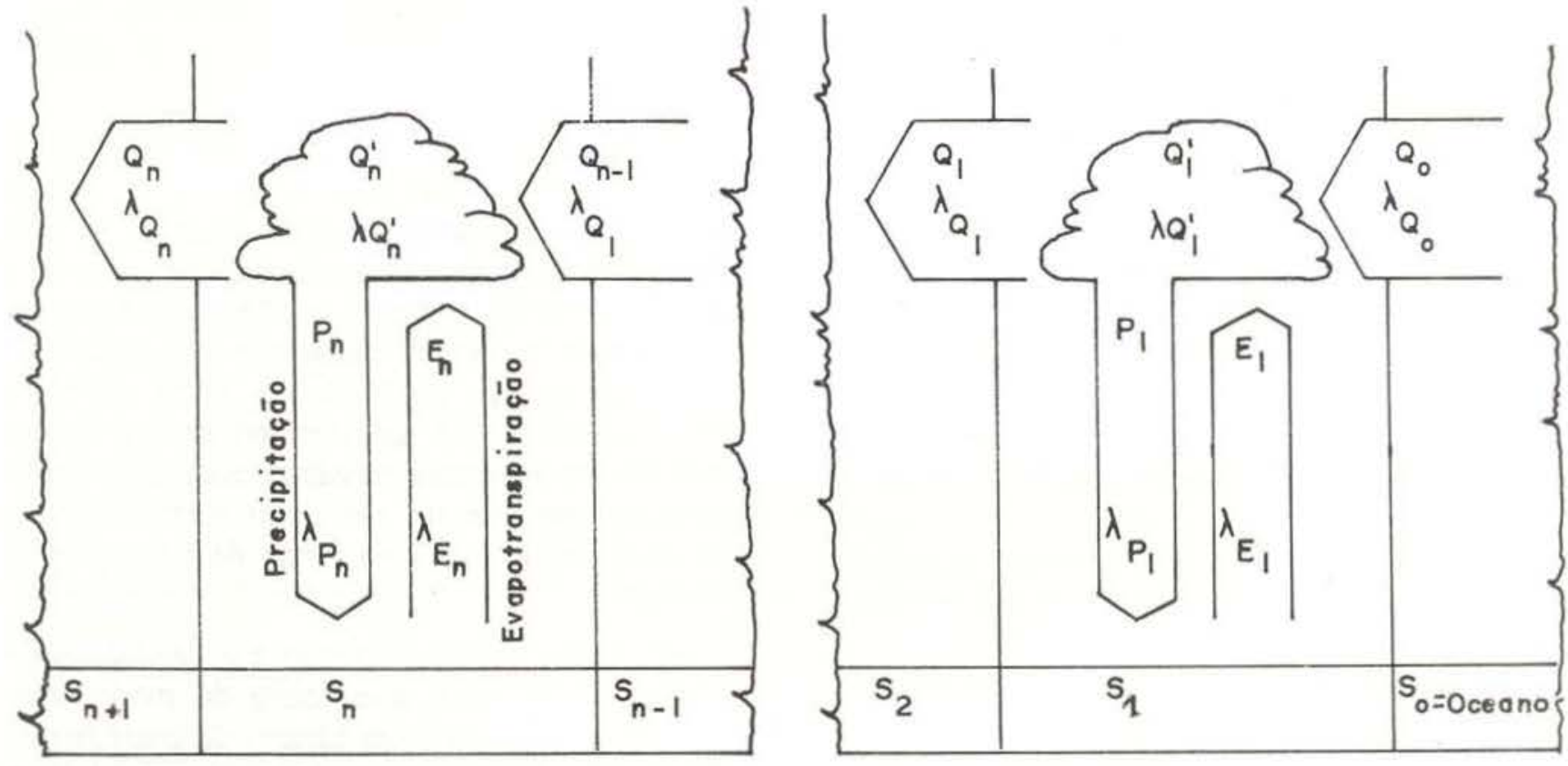

Fig. 3 - Modelo para o cálculo dos $\lambda_{\mathrm{C}_{\mathrm{n}}}$ incluindo os efeitos da uvapotranspiraçāo.

Resulta, portanto, que o enriquecimento isotópico, relativo ao V-SMOW, das precipitações $\mathrm{P}_{\mathrm{n}}$ do setor $\mathrm{S} n$, será dado pela

$$
y_{P_{n}}=\frac{1-F_{n}^{\propto}}{1-F_{n}} y_{O_{n}}{ }^{\prime} \cdots \cdots \cdots \cdot
$$

onde a fração residual de vapor é agora estimada pela

$$
F_{n}=\frac{Q_{n}^{\prime}-P_{n}}{Q_{n}^{\prime}}=\frac{Q_{n-1}+E_{n}-P_{n}}{Q_{n-1}+E_{n}} \ldots
$$

Enfim, o enriquecimento isotópico, relativo a V-SMOW, do vapor residual $Q_{n+1}^{\prime}$, será dacio pela

$$
y_{Q^{\prime}}=\frac{F_{n}^{\propto C}}{F_{n}} y_{Q^{\prime}} \cdots \cdots \cdots .
$$

As relaçōes (10) e (12) são válidas na hipótese de que cada fonte de vapor contribua para as precipitações, proporcionalmente à massa de vapor correspondente ou, em outras palavras, que a condensação seja um processo independente da origem do vapor.

As relações (9), (10) e (12) permitem então, computar a composição isotópica das precipitações $\mathrm{P}_{\mathrm{n}}$ de cada setor $\mathrm{S}_{n}$ desde que sejam conhecidos, além dos parâmetros indicados no modelo de Rayleigh, os seguintes valores :

v. os totais de evapotranspiração média mensal, $E_{n}$, de cada setor $S_{n}$ e

vi. o enriquecimento isotópico relativo y $E_{n}$ do vapor originado pela evapotranspiração.

Como foi apresentado no capítulo Material e Método, só são disponiveis os níveis médios de evapotranspiração $\mathrm{En}_{\mathrm{n}}$, estimados pelo método de Penmann, adaptado à floresta de grande porte e extensão (Villa Nova, 1976) .

De outro lado, os valores de $y_{E_{n}}$ podem ser identificados com os valores do enriquecimento isotópico, relativo a V-SMOW, das próprias precipitaçōes $\mathrm{Pn}_{\mathrm{n}}$, pois :

a, o processo de evapotranspiração não induz fracionamento isotópico na água do sclo (Zimmermann, 1966) e, portan- 
to, a composição isotópica média do vapor originado por esse processo é igual àquele cia água absorvida pelas plantas;

b. estão sendo analisados vaiores médios mensais, enquanto $\sigma$ tempo de trânsito da água, associado ao processo de evapotranspiração é curto, relativamente ao período considerado.

Pode-se, portanto, admitir-se que a água evapotranspirada, durante um determinado mês, origina-se, predominantemente, das precipitaçōes do mês considerado.

Enfim, a composição isotópica do vapor de origem oceânica $\mathrm{O}_{0}$, é estimada a partir da composição isotópica das precipitações $\mathrm{P}_{1}$ do setor mais oriental, $\mathrm{S}_{1}$, de modo análogo ao modelo precedente. Pelas expressões (9) e (10), tem-se :

$$
\mathrm{y}_{\mathrm{Q}}=\frac{1-\mathrm{F}_{1}}{1-\mathrm{F}_{1}}\left(\mathrm{Q}_{0}+\mathrm{E}_{1}\right)-\mathrm{E}_{1} \frac{\mathrm{y}_{\mathrm{P}_{1}}}{\mathrm{Q}_{0}} \ldots
$$

\section{Resultados E DIscussão}

Introduzindo os dados das Tabelas 1,2 e 3, nas expressões (8) e (13) obtém-se as composições isotópicas do vapor oceânico reportados na Tabela 4; enquanto que, utilizando as expressōes (6') e (10), obtém-se os valores reportados na Tabela 5 . Tais resultados são expressos na forma de desvios relativos ao V-SMOW, definidos em material e método.

\section{COMPOSIÇÃO ISOTÓPICA DO VAPOR OCEÂNICO}

Como foi explicado no capítulo Teoria do Método, a falta de determinaçōes suficientemente representativas, da composição isotópica do vapor oceânico ao entrar na bacia, pode ser facilmente superada estimando tal composiçāo a partir das concentrações isotópicas médias das precipitações do setor mais oriental da bacia, $\mathrm{S}_{1}$.

Os valores de ${ }^{\delta} \mathrm{Q}_{0}$, reportados na Tabela 4 , juntamente com os valores médios $\&_{\mathrm{C}_{1}}$ utilizados, se apresentam sistematicamente mais negativos daqueles medidos no vapor do Atlân- tico Norte por Graig \& Gordon (1965) que apresentavam um intervalo de variação para $\delta$ entre $-9,6$ e $-11,3^{\circ} / 00$; contudo, o vapor em questão foi coletado à altura do mastro princi. pa! do navio enquanto os valores de ${ }^{\delta} \mathrm{Q}_{\mathrm{O}}$ pretendem representar a composição média da massa total de vapor: a contribuição das camadas superiores, sempre mais leves, tenderia justamente a fornecer valores de ${ }^{\delta} \mathrm{O}_{0}$ mais negativos.

Além disto, valores mais negativos de ${ }^{\delta} \mathrm{O}_{0}$ podem também ser devidos ao fato de que a massa de vapor foi já sujeita, antes de entrar na bacia, a algumas precipitações que teriam deixado o vapor residual empobrecido em isótopos mais pesados.

Enfim, a estimativa de ${ }^{\mathcal{\delta}} \mathrm{Q}_{0}$ utilizada dá um peso, provavelmente excessivo, às estações da costa. (liha de Marajó e Belém) pela ausência de um número suficiente de estações de coleta no interior do setor e pode, purtanto, acontecer que, em cieterminados meses, tal estimativa não seja suficientemente representativa.

Analisando as relaçōes fornecidas pelos modelos, verifica-se que os valores dos $\delta_{C_{n}}$ são linearmente dependentes de $\delta_{\mathrm{O}_{0}}$ e que, pcrtanto, uma variação na sua estimativa determina um simples deslocamento, positivo ou negativo, dos valores de ${ }^{\delta} \mathrm{C}_{\mathrm{n}}$

TABELA 4 - Enriquecimento isotópico ponderado das precipitações do setor $\mathrm{S}_{1},{ }^{-} \mathrm{SC}_{1}$, e do vapor de origem oceânica $8 Q_{\circ}(e m \%)$, em considerar o efeito da evapotranspiração (a) e incluindo a evapotranspiração (b).

\begin{tabular}{lccc}
\hline \multirow{2}{*}{ Mês } & & \multicolumn{2}{c}{$\delta Q_{0}$} \\
\cline { 3 - 4 } & $\delta C_{1}$ & & $b$ \\
\cline { 3 - 4 } & & $-15,2$ & $-16,0$ \\
JAN & $-6,4$ & $-14,2$ & $-14,7$ \\
FEV & $-5,0$ & $-14,0$ & $-14,6$ \\
MAR & $-4,7$ & $-13,7$ & $-14,2$ \\
ABR & $-4,6$ & $-15,5$ & $-15,5$ \\
MAI & $-6,1$ & $-12,6$ & $-13,2$ \\
JUN & $-3,9$ & $-11,4$ & $-12,0$ \\
JUL & $-2,1$ & $-11,1$ & -11.8 \\
AGO & $-1,9$ & $-10,8$ & $-11,5$ \\
SET & $-1,5$ & $-10,5$ & $-11,3$ \\
OUT & $-1,1$ & $-9,8$ & $-10,5$ \\
NOV & $-0,4$ & $-11,3$ & $-11,9$ \\
DEZ & $-2,1$ & & \\
- & & &
\end{tabular}


A VARIAÇÃO DA COMPOSIÇÃO ISOTÓPICA AO LONGO DA BACIA

Pelos resultados da Tabela 5 , o modelo, baseado numa única fonte de vapor, mostra uma variação, ao longo da bacia, monotônica e decrescente, sendo a derivada de $\varsigma_{C}$, relativa à latitude no sentido E-W, sempre positiva e crescente, em todos os períodos em que o modelo é aplicável.

Os dados experimentais, de outro lado, in dicam uma variabilidade mais complexa que vai desde uma marcada constância, até a indicação da existência de um máximo ou mínimo na parte central da bacia. Mesmo quando 03 dados experimentais variam de modo monotônico decrescente ao longo da bacia, tal variaçāo é, na maioria dos casos, significativamente inferior a aquele previsto pelo modelo, como pode-se observar na Figura 4 , referente ao mês de dezembro.
A inclusāo da evapotranspiração, como fonte de vapor, além de constituir um modelo aplicável a todos os meses do ano, tende a fornecer um melhor ajuste com os dados ex. perimentais, como pode ser observado na Fi. gura 4, já citada. Em particular, sua introduçāo modifica apreciavelmente a forma da variação, da composição isotópica das precipitaçōes ao longo da bacia como se pode observar nos dados da Tabela 5; assim por exempio, no trimestre julho-agosto-setembro, o modelo fornece valores máximos para ${ }^{\delta} \mathrm{C}_{\mathrm{n}}$ correspondentes aos setores centrais da bacia, contrastando portanto, com a variação monotônica decrescente obtida quando se considera somente o vapor de origem oceânica.

Mesmo spresentando um melhor ajuste e refletindo melhor a variação da composição isotópica das precipitações ao longo da faixa

TABELA 5 - Valores médios mensais de $\delta_{18}$ (em \% computados sem (a) e com (b) a contribuiçāo da evapotranspiração. Nos meses de janeiro a maio nảo foi possivel fazer os cálculos pelo modelo (a) por nāo havevr suficiente va. por dágua na atmosfera, para originar as precipitaçōes neste período, admitindo-se como única fonte de vapor dágua o oceano Atlântico.

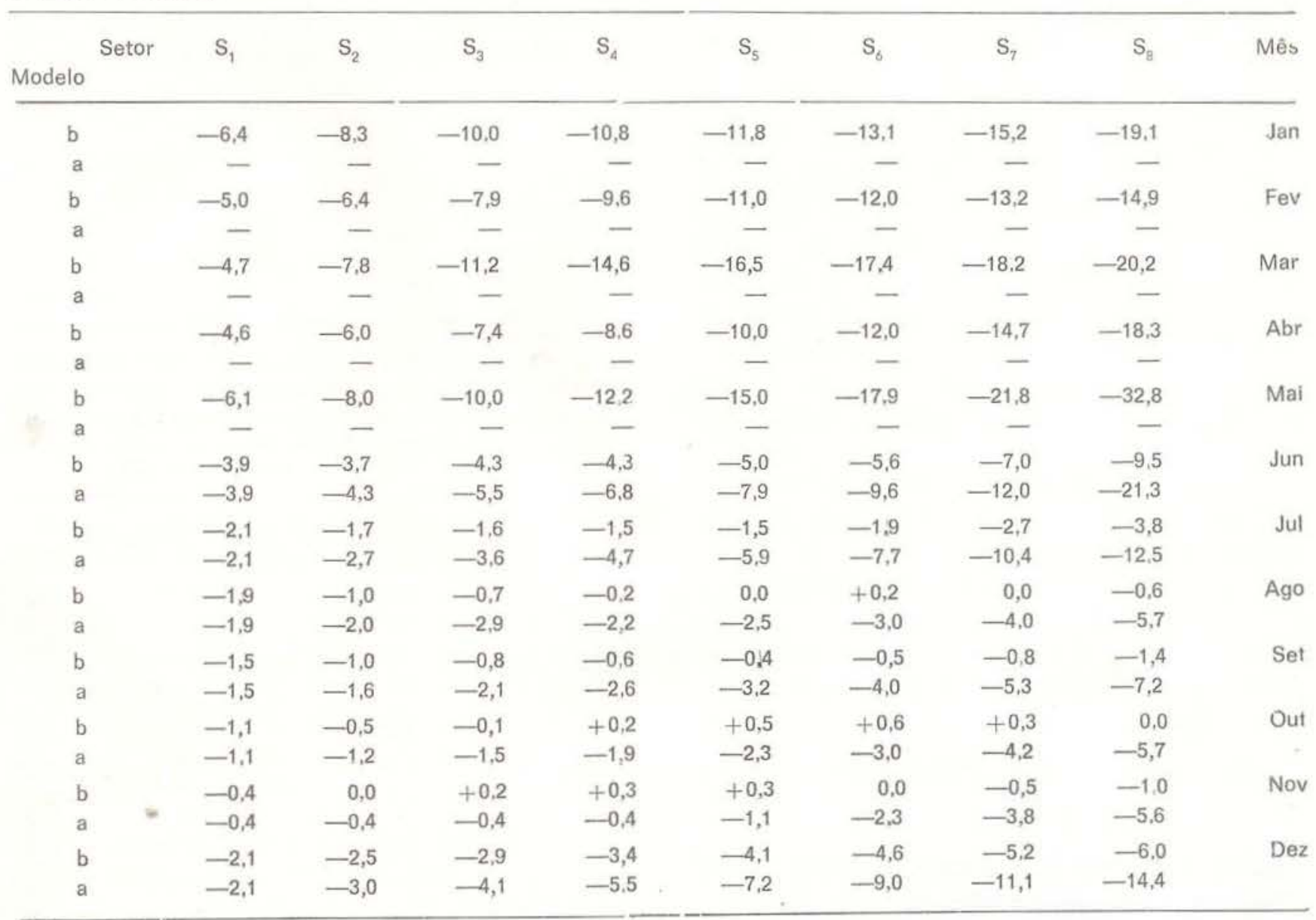


central da bacia, os valores calculados apresentam ainda diferenças significativas com re lação aos dados experimentais.

Tais diferenças podem ser atribuidas às hipóteses simplificadoras utilizadas, que se fazem necessárias face à ausência de determinações experimentais adequadas de vários parâmetros.

Assim, por exemplo, os níveis da evapo. transpiração não são valores específicos para o ano considerado (1974), mas săo valores médios estimados a partir dos valores médios de 10 anos.

Em particular, nos primeiros cinco meses de janeiro até maio, será necessário que a fonte de vapor de isótopos mais pesados, ou seja a evapotranspiraçấo, tenha niveis superiores aos utilizados neste trabalho. Com efeito, aumentando-se a evapotranspiração foi possível adaptar melhor os valores teóricos aos experimentais. A falta de dados meteorológi-
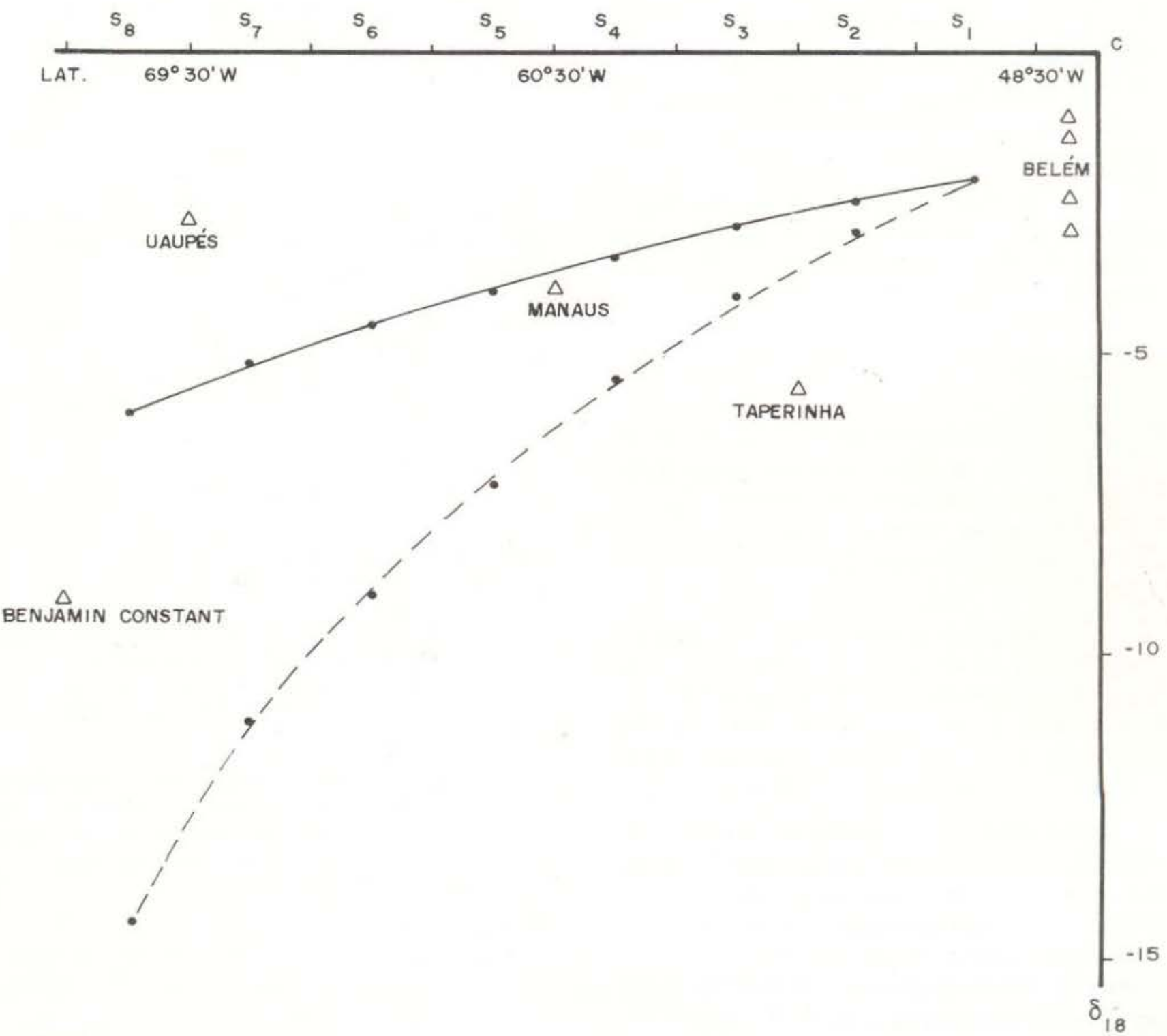

Fig. 4 - Comparaçâo dos dados isotópicos $\left(\S_{18}\right)$ esperados, para água de chuva, nos setores $\mathrm{S}_{1}$ a $\mathrm{S}_{8,}$ a partir dos modelos considerando (-) e não considerando (-..) a contribuição da evapotranspiração e os valores encontra$\operatorname{dos}(\Delta)$ experimentalmente. 
cos não permite estimar com maior precisão os fluxos meridionais de vapor dágua que poderiam eventualmente influenciar no cálculo da composição isotópica, mesmo se sua contribuição ao balanço de massa for desprezíve! Em média foi necessário admitir-se que as fontes de vapor (Oceano e Floresta) são da mes. ma ordem de grandeza, o que indica uma con. tribuição às precipitações da ordem de $50 \%$ pela evapotranspiração.

Deve-se salientar ainda (Molion, 1976) que possivelmente o vapor dágua na parte mais baixa da atmosfera tem um ciclo mais rápido que o vapor das altas camádas envolvi. do no processo da formação das chuvas.

Esse efeito deverá ser levado em consideração em modelos mais detalhados sobre o frácionamento isotópico da região.

Uma análise crítica dos dados mensais e um estudo de médias trimestrais e anuais será objeto de outro trabalho (Salati et al., em preparação) .

\section{CONCLUSÕES}

O método isotópico mostrou-se útil no estudo do ciclo da água na região Amazônica. Pode-se tirar conclusões por método indepen dente, além de permitir uma compreensão mais detalhada da recirculação da água na re. gião.

Em média, foi necessário admitir-se que a contribuiçãc do vapor dágua gerado pelas plantas foi da ordem de $50 \%$ da precipitação. Desta maneira a contribuição do vapor gerado dentro da região é da mesma ordem de grandeza que o vapor proveniente do oceano.

Serão necessárias determinações mais detalhadas, especialmente a composição isotópica do vapor atmosférico, a fim de se poder estimar com melhor precisão a influêricia dc vapor gerado pela transpiração das plantas. É possível, através deste método, estimar a pró. pria evapotranspiração real da floresta.

Será também conveniente aumentar o número de pontos de observação da composição isotópica, a fim de se poder melhor testar o modelo proposto.
A utilização desta metodologia, associada ao estudo do movimento das massas de ar através de radiossondagem, poderá trazer um entendimento mellhor da dinâmica da atmosfera na regiăo.

\section{AGRADECIMENTOS}

Este trabalho foi realizado com o apoio financeiro da CNEN, FINEP e CNPq. Os autores agradecem a colaboração do IDESP através de seus pesquisadores Carlos Moura dos Reis e Antonio Carlos F.N.S. Tancredi e do INPA através de sua pesquisadora Maria de Nazaré Góes Ribeirn.

\section{SUMMARY}

Two models on the isotopic fracteonation of water are presented in this paper. In the first model, it is assumed that the only source of water vapour for the Amazon region is the Atlantic Ocean, introduced by the predominant easterly winds. The second model contains the assumption that the forest also serves as a source of water vapour contributing an equal volume of water to the regional rains as the vapour of oceanic origin.

\section{BIBLIOgRafia}

Craig, H. \& Gordon, L.I.

1965 - Isotopic Oceanography: Deuterım and Oxygen-18 variations in the Ocean and the Marine Atmosphere. In: Tongiorgi, E. ed. Stable Isotopes in Oceanographic Studies Studies and Paleotemperatures. Lab. Div. Geologia Nucleare, Pisa, pp. 9-1360.

Dall'olio, A.

1976 - A composição isotópica das precipitaçōes do Brasil: modelos isotérmicos $\mathrm{e}$ a influência da evapotranspiraçăo na Bacia Amazônica. Dissertação de Mestrado, ESALQ/USP, $180 \mathrm{p}$.

GONFIANTINI, R.

1978 - Standards for stable isotope measurements in natural compounds. Nature, 271(5645) : 536 .

Marques, J.; Santos, J.M.; Villa Nova, N.A. \& SALATI, E.

1977 - Precipitable water and water vapor flux between Belém and Manaus. Acta Amazonica, $7(3): 335-362$. 
Matsui, E.; Salati, E.; Brinkmann, W.L.F. \& FRIEDMAN, I.

1972 - Vazões relativas dos rios Negros e Solimões através das concentrações de 180 . Acta Amazonica, 3(2) : 31-46.

Matsui, E.; Salati, E.; Friedman, I. \& Brinkmann, W.L.F.

1976 - Isotopic hydrology in the Amazonia 2. Relative discharges of the Negro and Solimões rivers through 180 concentrations. Water Resources Research, 12(4) : 781-785.

MoLion, L.C.B.

1976 - A climatonomic study of the energy and moisture fluxes of the Amazonas basin with considerations of deforestation effects. Tese de PHD, Universidade de Visconsin-Madison, $133 \mathrm{p}$.

ReIS, C.M.; TANCREdi, A.C.F.N.S.; MatsuI, E. \& SALATI, E.

1977 - Caracterização das águas da região de Marajó através de concentraçōes de 180 e D. Acta Amazonica, 7(2): 209-22.
Salati, E.; Dallólio, A.; Matsui, E. \& Gat, J.R. 1979 - Recycling of water in the Amazon river an isotopic study. Water Resources Research (no prelo).

Salati, e.; Matsui, E.; Cervellini, A. \& Friedman, i. 1979 - Concentração de 180 e D, em águas fluviais da Amazônia, Boletim do CENA (no prelo).

Villa Nova, N.A.: Salati, E. \& Matsui, E.

1976 - Estimativa da evapotranspiração ra bacia Amazônica. Acta Amazonica, Manaus, 6(2) : 215-228.

Zimmermann, U.; EhHalt, D. \& Munnich, K.O.

1966 - Soil water movement and evapotranspiration: changes in the isotopic composition of the water. In: Symposium of Isotopes in Hydrology. IAEA, Vienna, 14 a 18 de novembro de 1966.

(Aceito para publicação em 8/11/79) 